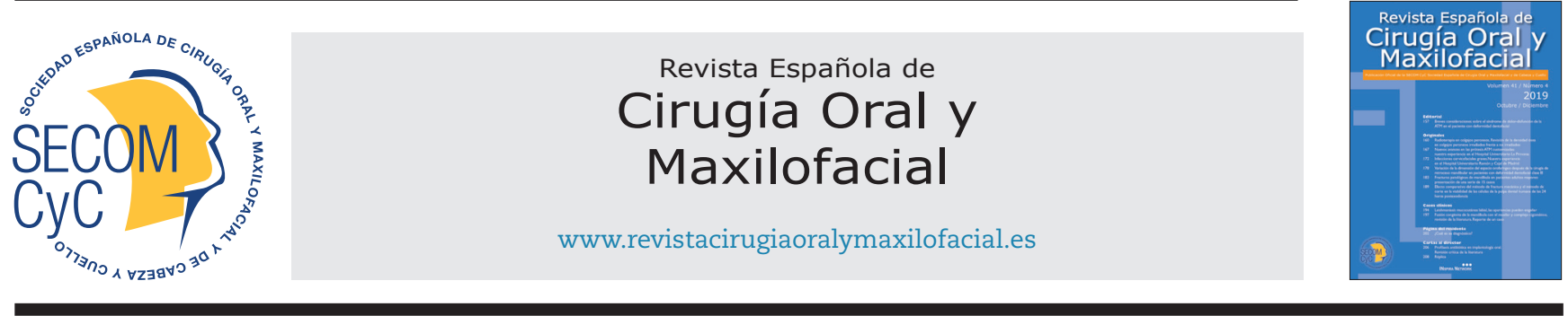

\title{
Original
}

\section{Nuevos avances en las prótesis ATM customizadas: nuestra experiencia en el Hospital Universitario La Princesa}

\section{Philip J. Brabyn ${ }^{a *}$, Francisco José Rodríguez Campo ${ }^{b}$, Verónica Escorial ${ }^{b}$, Mario Fernando Muñoz-Guerra ${ }^{b}$, Daniel Pastor Zuazaga ${ }^{c}$ y Fernando Valiente Infantec}

${ }^{a}$ Médico residente. Servicio de Cirugía Oral y Maxilofacial. Hospital Universitario La Princesa, Madrid, España. ${ }^{b}$ Médico. Servicio de Cirugía Oral y Maxilofacial. Hospital Universitario La Princesa, Madrid, España. 'Médico. Servicio de Cirugía Oral y Maxilofacial. Hospital Sant Joan d'Alacant, Alicante, España

INFORMACIÓN DEL ARTÍCULO

\section{Historia del artículo:}

Recibido el 4 de julio de 2019

Aceptado el 11 de octubre de 2019

Palabras clave:

Articulación temporomandibular, reconstrucción ATM, prótesis ATM.

\section{R E S U M E N}

Introducción: Presentamos nuestra experiencia y serie de casos en los que se ha utilizado la prótesis de ATM diseñada y desarrollada en España por el Dr. Carlos Goizueta (Hospital Sant Joan d'Alacant) junto con el Instituto Tecnológico de Canarias y Maxilaria.

Materiales y métodos: Pacientes tratados con patología de la articulación temporomandibular y que han sido reconstruidos con una prótesis de ATM. Esta prótesis tiene cuatro componentes, dos de la fosa articular (una parte de titanio y la otra de polietileno) y dos del cuerpo de la prótesis (cabeza de cromo-cobalto y rama de titanio).

Resultados: Se presentan los resultados preliminares de los pacientes reconstruidos con prótesis de ATM en los últimos 2 años en nuestro centro. Se han colocado un total de 8 prótesis en 6 pacientes. El diagnóstico de los pacientes fue anquilosis en 4 de los casos, y disfunción ATM avanzada en los otros 2. Se vio una mejoría, tanto del dolor como de la apertura oral, en un seguimiento mínimo de 6 meses.

Conclusión: Esta prótesis de ATM, de desarrollo nacional, tiene varias ventajas como son la posibilidad de recambio de algún componente y también el diseño del mismo hace que las fuerzas masticatorias se transmiten a la rama mandibular y no a los tornillos de la prótesis.

\footnotetext{
*Autor para correspondencia:

Correo electrónico: philipbrabyn@gmail.com (Philip J. Brabyn).
}

DOI: 10.20986/recom.2019.1082/2019

1130-0558/@ 2019 SECOM. Publicado por Inspira Network. Este es un artículo Open Access bajo la licencia CC BY-NC-ND (http:// creativecommons.org/licenses/by-nc-nd/4.0/). 


\section{New advances in customized TMJ prosthesis: our experience at La Princesa University Hospital}

\section{A B S T R A C T}

Keywords:

Temporomandibular joint, TMJ reconstruction, TMJ prosthesis. together with the Technological Institute of Canarias and Maxilaria
Introduction: We present our experience and case series in which we used a TMJ prosthesis designed and developed in Spain by Dr. Carlos Goizueta (Hospital Sant Joan d'Alacant),

Materials and methods: Patients diagnosed with temporomandibular joint pathology who were reconstructed with a TMJ prosthesis. This prosthesis has four components, two of the articular fossa (one part of titanium and the other part of polyethylene) and two of the body of the device (condylar head of cobalt-chrome and ramus of titanium).

Results: We present the preliminary results of the patients reconstructed with a TMJ prosthesis in the past 2 years in our center. A total of 8 prosthesis were place in 6 patients. The diagnosis of the patients was TMJ ankylosis in 4 cases, and severe TMJ dysfunction in the other 2 cases. An improvement of both pain and also mouth opening was observed after a follow-up of at least 6 months.

Conclusion: This TMJ prosthesis, developed in Spain, has many advantages, such as the possibility of replacement of some of the parts, as well as its design since the masticatory forces are transmitted to the mandibular ramus and not to the screws of the device.

\section{INTRODUCCIÓN}

La reconstrucción alogénica de la articulación temporomandibular (ATM) es la sustitución de una articulación que presenta un daño irreparable ${ }^{1}$. Los objetivos de esta reconstrucción son restaurar la morfología articular, restaurar la longitud de la rama ascendente, aumentar el rango de movimiento articular y mejorar la oclusión del paciente. Este tipo de reconstrucción está indicado en patología severa de la ATM, entre las que se incluyen los estadios avanzados de osteoartrosis, las secuelas traumáticas condilares, la anquilosis de ATM, las reabsorciones condilares y los tumores condilares, entre otros².

Entre las prótesis de ATM que se utilizan en España, existen de tipo stock y también customizadas. La mayoría de las prótesis tienen el componente del cuerpo mandibular de titanio, la cabeza del cóndilo de cromo-cobalto y la fosa puede ser metálica o de polietileno.

El objetivo del presente trabajo es presentar la experiencia de nuestro centro con el uso de una nueva prótesis de ATM customizada, diseñada y fabricada en España.

\section{MATERIALES Y MÉTODOS}

La prótesis que se presenta es una prótesis completamente customizada CAD/CAM con varios componentes. La rama mandibular es de titanio, y se fija al hueso mediante tornillos, también de titanio. La cabeza condilar es de una aleación de cromo-cobalto, tiene una forma redondeada y se encaja a presión sobre la parte superior del componente mandibular. La fosa tiene dos componentes, un componente de titanio que se fija mediante tornillos a la eminencia (junto con una fosa de titanio poroso), y otra parte que contacta con el cóndilo protésico de polietileno de alto peso molecular (UHMWPE) (Figura 1). Fue diseñada y desarrollada inicialmente por el Dr. Goizueta (Hospital San Juan de Alicante), junto con el Instituto Tecnológico de Ca-

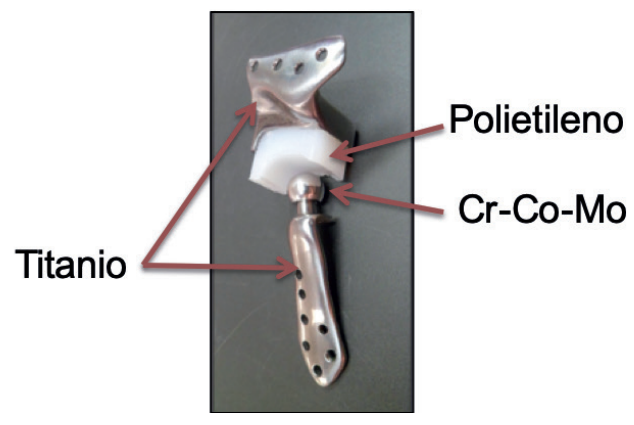

Figura 1. La prótesis de ATM con rama de titanio, cabeza de cromo-cobalto y fosa de polietileno de alto peso molecular y de titanio.

narias y Maxilaria y, tras su fallecimiento, continuaron su desarrollo el Dr. Valiente (de la misma institución) y el Dr. Rodríguez Campo (Hospital Universitario La Princesa).

En este estudio preliminar se recogieron los pacientes tratados de su patología de la ATM mediante una reconstrucción con la prótesis presentada. Las dos variables clínicas analizadas fueron la máxima apertura oral (MAO, medida en milímetros) y el dolor (medido mediante escala visual, VAS: 0-100) previos a la intervención y al mes, 3 y 6 meses del postoperatorio. Se utilizó la prueba T de Student para muestras apareadas en el análisis, considerando una p < 0,05 como el umbral de significación estadística.

\section{Técnica quirúrgica}

Previa a la intervención, se realiza una planificación y un diseño CAD/CAM de la prótesis incluyendo unas guías de corte para realizar las osteotomías y unos comprobadores de espacio para la correcta colocación de la prótesis (Figura 2). La 


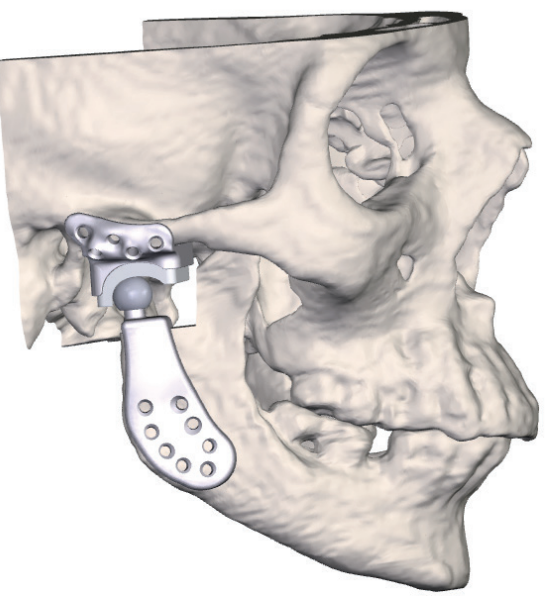

Figura 2. Planificación y diseño CAD/CAM de la prótesis.

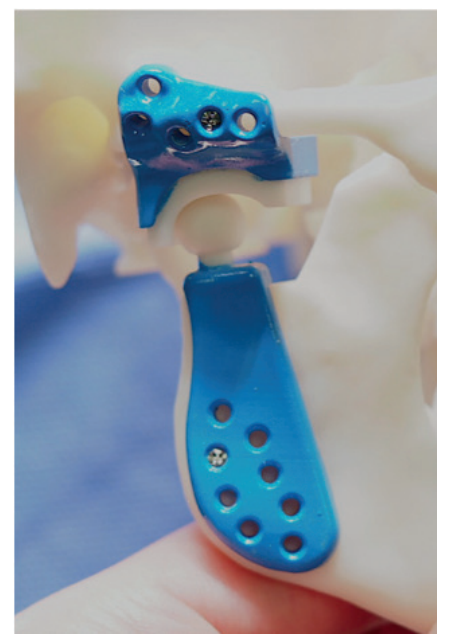

Figura 4. Modelo esterolitográfico con el modelo de la prótesis. cirugía se realiza, como suele ser habitual, mediante una incisión preauricular con extensión temporal y una segunda incisión retromandibular. Al alcanzar la cápsula articular, esta se abre para exponer la articulación. Se realizan las osteotomías con ayuda de guías de corte. Primero, se realiza una condilectomía y retirada del menisco articular (si existe) para poder realizar posteriormente una osteotomía de la rama mandibular según la guía de corte. En la fosa-eminencia se realiza un curetaje de las estructuras fibrocartilaginosas para conseguir una buena adaptación del componente craneal de la prótesis; en ocasiones es necesario realizar una ligera osteotomía. Posteriormente, tras colocar un bloqueo intermaxilar (BIM), se comprueba la adaptabilidad de los componentes craneal y mandibular, así como el espacio disponible mediante distintos comprobadores (Figura 3). Por último, se colocan las prótesis definitivas, fijándolas parcialmente. Se retira el BIM, se comprueba la funcionalidad de las prótesis y, si todo es correcto, se completa la fijación de las prótesis, no siendo necesario ya mantener el BIM (Figura 4).

De forma habitual, se obtiene grasa autóloga de la zona periumbilical y se coloca alrededor de las prótesis para evitar

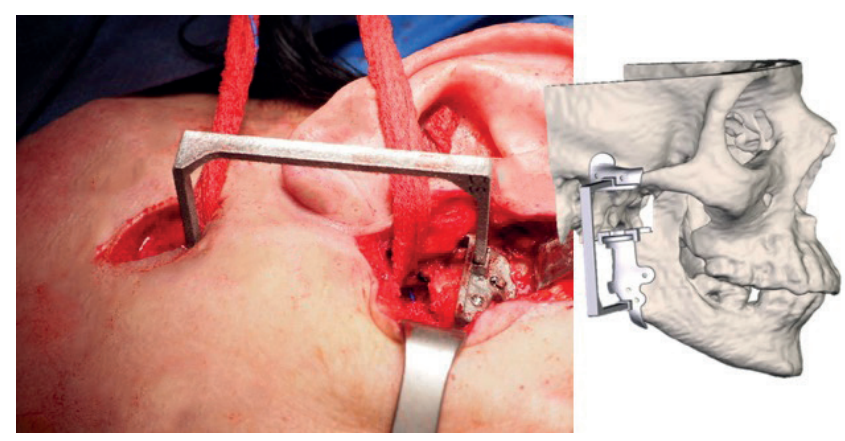

Figura 3. Imagen del comprobador de espacio durante el procedimiento quirúrgico y en la planificación. o reducir el espacio muerto tras las osteotomías. La realización de una coronoidectomía dependerá de cada caso, aunque es habitual en las anquilosis. No se deja ningún drenaje.

Todos los pacientes recibieron una profilaxis antibiótica con amoxicilina/ácido clavulánico $1 \mathrm{~g}$ i.v. cada $8 \mathrm{~h}$ durante el ingreso, pasando a vía oral al alta hasta cumplir ciclo de 5 días (en los casos de alergia a penicilinas, la profilaxis se realizó con clindamicina $300 \mathrm{mg}$ cada $8 \mathrm{~h}$ ). En el postoperatorio inmediato, y durante las 3-4 primeras semanas, los pacientes siguieron un protocolo de dieta blanda y unos ejercicios de fisioterapia mandibular (ejercicios de apertura, cierre y movilidad mandibular).

\section{RESULTADOS}

Se presentan los resultados preliminares de los pacientes reconstruidos con prótesis de ATM customizada desde el año 2017 en nuestro centro, con un seguimiento mínimo de 6 meses. Se han colocado un total de 8 prótesis en 6 pacientes mujeres. La edad media al diagnóstico fue de 44,8 años (26-69). El diagnóstico de las pacientes fue: a) anquilosis en 3 casos, y b) disfunción ATM avanzada (grado V Wilkes) en 2 casos; una paciente con una afectación bilateral presentaba anquilosis en un lado y disfunción grado V Wilkes en el otro lado. Las 6 pacientes habían sido intervenidas previamente de la articulación afecta, ya sea mediante artroscopia (2 casos), artroplastia abierta (1 caso) o ambos procedimientos (en 3 casos). En dos casos la afectación fue derecha, en dos izquierda, y en otros dos bilateral.

En el punto de control postoperatorio a los 6 meses se objetivó un incremento medio de la MAO de $13 \mathrm{~mm}$, de 15,6 7,3 $\mathrm{mm}$ a $28,6 \pm 4,2 \mathrm{~mm}$, estadísticamente significativo ( $p=0,03)$. Igualmente se observó una reducción del dolor (VAS) de 61,6 \pm 30,7 a $21,6 \pm 27,9$, también estadísticamente significativo $(p=0,03)$.

No hubo fracaso de ninguna de las prótesis y solo hubo complicaciones leves en dos pacientes (paresia del nervio facial transitoria). Todas las prótesis permanecen estables y con buenos controles al final del seguimiento (Tabla I). 


\begin{tabular}{|c|c|c|c|c|c|c|c|c|c|c|}
\hline & Edad & Sexo & Cirugías previas & Diagnóstico & $\begin{array}{l}\text { Lado } \\
\text { prótesis }\end{array}$ & Complicaciones & $\begin{array}{c}\text { MAO } \\
(\mathrm{mm}) \\
\text { Pre }\end{array}$ & $\begin{array}{l}\text { MAO } \\
6 \mathrm{~m}\end{array}$ & $\begin{array}{l}\text { DOLOR } \\
\text { (VAS) } \\
\text { Pre }\end{array}$ & $\begin{array}{l}\text { DOLOR } \\
6 \mathrm{~m}\end{array}$ \\
\hline 1 & 47 & $\mathrm{~F}$ & Artroscopia & Anquilosis & Derecho & $\begin{array}{l}\text { Paresia VII } \\
\text { transitoria }\end{array}$ & 16 & 34 & 70 & 0 \\
\hline 2 & 47 & $\mathrm{~F}$ & $\begin{array}{l}\text { Artroscopia } \\
\text { Artrotomía (injerto } \\
\text { costocondral) } \\
\text { Prótesis bilateral* }\end{array}$ & $\begin{array}{c}\text { Disfunción V Wilkes } \\
\text { Anquilosis }\end{array}$ & Bilateral & No & 16 & 34 & 90 & 80 \\
\hline 3 & 44 & $\mathrm{~F}$ & $\begin{array}{c}\text { Artroscopia } \\
\text { Artrotomía } \\
\text { (meniscectomía) }\end{array}$ & Disfunción V Wilkes & Derecho & $\begin{array}{l}\text { Paresia VII } \\
\text { transitoria }\end{array}$ & 22 & 24 & 90 & 10 \\
\hline 4 & 69 & $\mathrm{~F}$ & Artroscopia & Anquilosis & Bilateral & No & 0 & 30 & 0 & 0 \\
\hline 5 & 36 & $\mathrm{~F}$ & Artrotomía & Disfunción V Wilkes & Izquierdo & No & 20 & 26 & 50 & 30 \\
\hline 6 & 26 & $\mathrm{~F}$ & $\begin{array}{l}\text { Artroscopia } \\
\text { Artrotomía }\end{array}$ & Anquilosis & Izquierdo & No & 20 & 24 & 70 & 10 \\
\hline
\end{tabular}

${ }^{*}$ Prótesis de otro sistema, que fue retirada por alergia a sus componentes.

\section{DISCUSIÓN}

Actualmente, existen tres tipos de prótesis de ATM, con diferentes componentes y formas de colocación. La prótesis de ATM desarrollada por Christensen ${ }^{3-5}$ es una prótesis completamente metálica (TMJ Implants/Nexus $\mathrm{CMF}^{\circledR}$, Salt Lake City, Utah, EE. UU.). La desventaja de esta prótesis es el problema relacionado con la fricción entre las partes metálicas (titanio y cromo cobalto), que puede llegar a desprender partículas tóxicas para el organismo.

Quinn desarrolló una prótesis (Lorenz/Zimmer Biomet ${ }^{\circledR}$, Warsaw, Indiana, EE. UU.) cambiando la fosa de titanio por una fosa de polietileno de alto peso molecular, aumentando el grosor de la fosa, lo que desciende el punto de rotación y permite una ganancia de apertura del 15-18 \% ${ }^{2,6}$. En un estudio con un seguimiento a largo plazo de más de 10 años $^{7}$ se demostró una mejoría clínica mantenida en el tiempo con el uso de esta prótesis.

Wolford desarrolló una prótesis customizada (TMJ Concepts $^{\circledR}$, Ventura, California, EE. UU.) con la fosa también de polietileno, pero con un componente de titanio para fijarla a la eminencia ${ }^{8-10}$. Describió el uso de la grasa autóloga sobre la prótesis, y su efecto sobre la mejoría de la apertura oral, la disminución del dolor postoperatorio y la disminución de crecimiento de hueso heterotópico ${ }^{11}$. Existen estudios ${ }^{12}$ de seguimiento a largo plazo tras la colocación de esta prótesis: el de mayor tiempo es de 20 años ${ }^{13}$, donde se describió una estabilidad a largo plazo en 56 pacientes. Ninguna de las prótesis tuvo que ser retirada por desgaste, y hubo una mejoría estadísticamente significativa tanto de la calidad de vida subjetiva del paciente como de la funcionalidad mandibular.

En 2003, Wolford y cols. ${ }^{14}$ publicaron una comparación entre la prótesis que habían desarrollado y la prótesis de Christensen. Describieron una mejoría de la MAO (aumento de 9,9 mm us. $6,7 \mathrm{~mm}$ ) y una mayor disminución del dolor (reducción del $43 \%$ us. el $23 \%$ ) con respecto a la prótesis de Christensen. En 2017, Johnson y cols. ${ }^{15}$ publicaron un metanálisis comparando los tres tipos de prótesis disponibles, en un total de 1165 pacientes. Sus resultados demostraron que la mejoría del dolor postoperatorio y de la MAO eran comparables entre los tres tipos, pero no había datos suficientes para comparar las tasas de fracaso y complicaciones entre los diferentes tipos.

La prótesis de ITC/Maxiliaria ${ }^{\circledR}$ (Madrid, España) es una nueva prótesis de ATM completamente customizada que tiene varias ventajas frente a las otras prótesis comercializadas en España. Igual que las prótesis desarrolladas por Quinn y Wolford, se utiliza una fosa de polietileno que tiene un cierto grosor (aprox. $4 \mathrm{~mm}$ ) que desciende el punto de rotación consiguiendo así una mayor apertura oral al conseguir no solo una rotación sino también una "pseudo-traslación" mandibular permitiendo la actuación del músculo pterigoideo lateral funcional del lado contralateral. Existe la posibilidad de recambio de alguno de sus componentes por posible desgaste, como la cabeza del cóndilo o el componente de polietileno, sin tener que recambiar la prótesis completamente con las complicaciones que eso conllevaría. La utilización de guías de corte y de comprobadores de corte y del espacio disponible hace que su colocación sea sumamente precisa. El diseño de la parte alta del componente mandibular hace que el cóndilo apoye directamente en la osteotomía mandibular, de forma que teóricamente las fuerzas masticatorias se transmiten directamente a la rama y no a los tornillos de la prótesis.

El presente estudio es un trabajo de resultados preliminar de una serie corta, donde se expone la técnica y el uso de esta prótesis, objetivando la mejoría clínica de los pacientes y la ausencia de complicaciones intraoperatorias y durante el postoperatorio inmediato. Sin embargo, la ausencia de un seguimiento superior a dos años para ningún paciente imposibilita la consecución de conclusiones firmes con respecto a estabilidad y posibles complicaciones a largo plazo. No se ha podido realizar ningún estudio comparativo con las otras prótesis utilizadas, ya que solo se ha utilizado una prótesis customizada de otro tipo en 2 ocasiones, y el resto han sido tipo stock. Otra limitación de este trabajo es que las ventajas que se exponen son hipotéticas y están basadas en la experiencia de los autores y no en un estudio experimental. 


\section{CONCLUSIÓN}

La prótesis de ATM presentada es una prótesis customizada de desarrollo nacional, con partes recambiables y cuyo diseño hipotéticamente permite una adaptabilidad precisa con una transmisión directa de las fuerzas masticatorias a la rama mandibular, mediante el apoyo directo del cóndilo protésico sobre la osteotomía mandibular y no sobre los tornillos. Los pacientes han mejorado en términos de dolor y apertura oral, manteniéndose las prótesis estables durante el corto periodo de seguimiento. Son necesarios estudios de series clínicas mayores y a más largo plazo.

\section{CONFLICTO DE INTERESES}

Los autores declaran no tener ningún conflicto de intereses.

\section{B I B L I O G R A F Í A}

1. Mercuri LG. Alloplastic temporomandibular joint reconstruction. Oral Surg Oral Med Oral Pathol. 1988;85(6):631-7. DOI: 10.1016/ S1079-2104(98)90028-2.

2. Quinn PD. Lorenz prothesis. Oral Maxillofacial Clin North Am. 2000;12(1):93-104.

3. Christensen RW. The correction of mandibular ankylosis by arthroplasty and insertion of a vitallium glenoid fossa prothesis: a new technique. Am J Ortho. 1963;5:16-24.

4. Russell R, Christensen R, Curry J, et al. Total joint replacement with alloplastic prosthesis. J Dent Res 1993;72:252.

5. Chase DC, Hudson JW, Gerard DA, Russell R, Chambers K, Curry $J R$, et al. The Christensen prosthesis: a retrospective clinical study. Oral Surg Oral Med Oral Pathol Oral Radiol Endod. 1995;80(3):273-8. DOI: 10.1016/s1079-2104(05)80382-8.
6. Macafee KA, Quinn PD. Total TMJ reconstruction with a Delrin titanium implant. J Craniofac Surg. 1992;3(3):160-9. DOI: 10.1097/00001665-199211000-00008.

7. Leandro LFL, Ono HY, de Souza Loureiro CC, Marinho K, Garcia Guevara HA. A ten-year experience and follow-up of three hundred patients fitted with the Biomet/ Lorenz Microfixation TMJ replacement system. Int J Oral Maxillofac Surg. 2013;42(8):100713. DOI: 10.1016/j.ijom.2013.04.018.

8. Wolford LM, Pitta MC, Reiche-Fischel O, Franco PF. TMJ Concepts/ Techmedica custom-made TMJ total joint prosthesis: 5-Year follow-up study. Int J Oral Maxillofac Surg. 2003;32(3):268-74. DOI: 10.1054/ijom.2002.0350.

9. Giannakopoulos HE, Sinn DP, Quinn PD. Biomet Microfixation Temporomandibular Joint Replacement System: A 3-year FollowUp Study of Patients Treated During 1995 to 2005. J Oral Maxillofac Surg. 2012;70(4):787-94. DOI: 10.1016/j.joms.2011.09.031.

10. Mercuri LG. Patient-Fitted ("Custom") Alloplastic Temporomandibular Joint Replacement Technique. Atlas Oral Maxillofac Surg Clin North Am. 2011;19(2):233-42. DOI: 10.1016/j.cxom.2011.05.001.

11. Wolford LM, Karras SC. Autologous fat transplantation around temporomandibular joint total joint prostheses: preliminary treatment outcomes. J Oral Maxillofac Surg. 1997;55(3):245-51. DOI: 10.1016/s0278-2391(97)90535-8.

12. Mercuri LG, Edibam NR, Giobbie-Hurder A. Fourteen-year followup of a patient-fitted total temporomandibular joint reconstruction system. J Oral Maxillofac Surg. 2007;65(6):1140-8. DOI: 10.1016/j.joms.2006.10.006.

13. Wolford LM, Mercuri LG, Schneiderman ED, Movahed R, Allen W. Twenty-Year Follow-up Study on a Patient-Fitted Temporomandibular Joint Prosthesis: The Techmedica/TMJ Concepts Device. J Oral Maxillofac Surg. 2015;73(5):952-60. DOI: 10.1016/j. joms.2014.10.032.

14. Wolford LM, Dingwerth DJ, Talwar RM, Pitta MC. Comparison of 2 temporomandibular joint total joint prosthesis systems. J Oral Maxillofac Surg. 2003;61(6):685-90. DOI: 10.1053/joms.2003.50112.

15. Johnson NR, Roberts MJ, Doi SA, Batstone MD. Total temporomandibular joint replacement prostheses: a systematic review and bias-adjusted meta-analysis. Int J Oral Maxillofac Surg. 2017;46(1):86-92. DOI: 10.1016/j.ijom.2016.08.022. 